\title{
Socioenvironmental Etiology of Plague Outbreak in Himachal Pradesh: A Retrospective Enquiry
}

\author{
Harvinder Kaur, Sonu Goel, Yachna Sharma, Rupali Raj Kessar, Amarjeet Singh
}

\section{ABSTRACT}

Aims: (1) To ascertain various socioepidemiological factors related to 2002 plague outbreak in Himachal Pradesh (HP). (2) To elucidate the sylvatic cycle of plague bacilli transmission.

Materials and methods: The study was conducted among affected families during 2002 HP plague outbreak. The opinion of natives of plague affected Hatkoti belt of HP, Gujjars and health care staff about etiology, proneness, sustenance, spread, control and prevention of plague were explored. Blood samples and fleas were collected from the domestic, peridomestic and wild rodents trapped from these areas. These were investigated entomologically and microbiologically in PGIMER, Chandigarh.

Results: Overall 159 rodents and 22 fleas were collected from wild and domestic areas. Three species of rodents were identified; Rattus rattus, Rattus norvegicus and Peromyscus leucopus. Similarly, three flea species namely Nosopsyllus fasciatus, Epitedia wenmanni and Leptopsylla segnis were extracted. Serum and blood samples $(n=49)$ collected from rodents showed Gram-positive bacilli (GPB), Gram-positive cocci (GPC) and diphtheroid bacilli.

Respondent villagers and Gujjars believed that plague outbreak occurred due to wrath of God and other supernatural forces. The villagers and health workers were of the view that exposure of people to plague was due to deep forest exploration for hunting.

Conclusion: The lifestyle of people and other social factors played a crucial role in plague outbreak in the area. The sylvatic cycle persisted in plague prone areas of HP.

Keywords: Fleas, Plague, Rodents, Socioepidemiology, Sylvatic cycle.

How to cite this article: Kaur H, Goel S, Sharma Y, Kessar RR, Singh A. Socioenvironmental Etiology of Plague Outbreak in Himachal Pradesh: A Retrospective Enquiry. J Postgrad Med Edu Res 2013;47(2):112-116.

\section{Source of support: Nil}

Conflict of interest: None declared

\section{INTRODUCTION}

Plague is a rapidly progressing zoonotic disease that is infamous for having caused the death of millions of people in large historic pandemics as well as numerous other deadly but localized outbreaks. It remains a significant global concern due to its persistence in many parts of the world, even in 21st century. It has also been touted as a weapon for bioterrorism and biological warfare. Whenever, there is a disruption of the social system, occurrence of natural disaster, or poor living conditions, plague is likely to break out.
Plague is primarily a disease of rodents and is maintained in wild rodent populations, through sylvatic cycle. ${ }^{1}$ Occasionally, it gets transmitted to commensal rats (urban plague) and then into human population. Infected commensal rats die in large numbers, as they have less resistance to the disease. The fleas living on them then seek alternative hosts. Y pestis taken up by the flea from an infected host in a blood meal undergoes a rapid multiplication in its stomach. The bacteria then form a viscous plug that blocks the stomach and prevents the flea from feeding normally. When the flea attempts to feed again, the blood comes up against the plug of bacteria and cannot enter the stomach. It is instead regurgitated by the flea back into the host. This process takes some bacteria with it, infecting the new host. ${ }^{2}$

The epidemics of plague in human beings originate when they come in contact with the infected rat fleas. Fleas inhabit infected rodents. Man enters only accidentally into the usual animal cycle. This cycle (rodent-flea-rodent) as a rule is enzootic (present in animal community at all times but occurring in only small number of cases). But under certain conditions, it reaches epizootic proportions (affecting many animals in any region at the same time).

There are three kinds of plague: Bubonic, primary pneumonic and septicemic, with the bubonic variety being the most common. The bubonic plague is characterized by swelling in groin. It is noncontagious. It is spread by a particular variety of rat flea. The dangerous pneumonic variety is characterized by infection in lungs and airway. It is highly infectious and spreads through personal contact. In septicemic plague, the blood is infected.

Natural foci of plague in India are known to exist in parts of Andhra Pradesh, Gujarat, Uttaranchal, Karnataka, Maharashtra, Tamil Nadu and Himachal Pradesh (HP). ${ }^{3}$ Recent plague outbreaks in India occurred in Beed (Maharashtra) and Surat (Gujarat) in 1994, followed by a local outbreak in HP in February, 2002 and a small outbreak in Uttaranchal in 2004. ${ }^{3}$ However, due to reasons unknown, an impression was created that plague ceases to exist in India after 1966. Despite being an internationally notifiable disease plague is not in the list of priority diseases by Government of India and other agencies. In addition, the disease is not given much importance in 
medical undergraduate and postgraduate teaching. In regions where clinicians are unfamiliar with plague, risk of misdiagnosis is high, and specific diagnostic tools are often not readily accessible in remote areas. Identification of the causal agent is critical for implementing immediate public health measures in the community. The present study is an attempt to study the socioepidemiological factors related to 2002 plague outbreak of HP. Simultaneously, efforts have been made to elucidate the sylvatic cycle of plague bacilli transmission in areas around Hatkoti region of $\mathrm{HP}$.

\section{MATERIALS AND METHODS}

This study is a part of an ICMR project sanctioned to the School of Public Health, Department of Community Medicine, PGIMER, Chandigarh. Before conducting the study, prior permission was obtained from DGHS (Directorate General Health Services) and Wild Life Authority of HP for field data collection. The study protocol was also approved by the Ethics Committee of the PGIMER, Chandigarh.

A total of five visits were made by the research team, each of 1 week duration, in remote and plague affected sites of HP between May 2011 and November 2011, where plague outbreaks had occurred in recent past. The places included Rohru, Hatkoti, Patsari, Mendali, Samala, Dodra, Dodra-Pujali, Seeladesh, Kotkhai, Mural Jungle, GariGanga Jungle, Parshal, Salayana, Nihari, Sarain Jungle, Deemo and Davas.

The opinion of natives, health care staff of the study area and Gujjar population (Muslim migrant shepherds) about various aspects of plague were explored through indepth interviews (IDI) and focus group discussions (FGD). A lifestyle profile of natives of affected villages was also created. The opinion of natives and Gujjars on repeated occurrence of plague in their area, role of other factors especially rats in spread of plague, type and pattern of disease, treatment seeking behavior, fear of disease in area, changes in health seeking behavior after 2002 outbreak, etc. were also catalogued. Available literature and reports on plague were also reviewed. Representatives of health care staff of each category (viz, doctors, pharmacists, laboratory technicians, nurses, ANMs) of the Community Development Blocks, i.e. Rohru, Jubbal, Nerua and Chaupal blocks were randomly selected and interviewed for their knowledge about etiology, proneness, sustenance, spread, control and prevention of plague, onguard measures adopted by them for plague, measures/criteria adopted for early detection of plague, home quarantine policy in case of plague suspect, modes of treatment and their treatment seeking behavior and other type of communicable diseases frequently encountered by them. Informed consent of respondents was duly taken.

During each visit, Sherman traps with bait were laid in domestic, peridomestic and wild areas of thick and dense vegetation. The traps were laid during dusk time and retrieved next day before the first light. After having made the assessment of trap positivity, the species of trapped rodents were identified. Combing method was used to screen rodents for ectoparasites. The blood samples of rodents (retro-orbital bleed) were collected. All the samples (rodent blood and rat fleas) were brought to PGIMER, Chandigarh, for further investigation and identification. They were stored in medical microbiology laboratory at optimum temperatures. These were investigated entomologically and microbiologically in PGIMER, Chandigarh.

A rapid serological test to identify F-1 antigen of Yersinia pestis in the rat/human serum samples was also done on 200 samples with crystal types Rapid Diagnostic Test (RDT) kit.

\section{RESULTS}

The study observed that the native people of HP earn their livelihood from agriculture, mainly apple orchards. They are highly superstitious having strong faith on their own 'Devi Devta' (local Gods). They visit 'Tantriks' (faith healers) for curing disease. Many local people are addicted to drugs also. Alcohol consumption and smoking are also common. The provision of medical facilities was not adequate. The agricultural area of villages in HP is attached to forest so peridomestic and wild rodents could be found inside houses in high densities. Hunting, though banned, is mostly done by them during winters in dense forests. They also regularly visit the caves in deep forests during their expeditions. It was emerged from the study that the index case acquired infection from these caves in 2002 plague outbreak. Therefore, it is highly probable that they were exposed to fleas in rats/burrows which led to higher spread of this disease. Some of the interviewed persons did know that plague is spread by rats through the bite of flea ('pissu') living on rat's body, while majority considered it a wrath of God and other supernatural forces.

The Gujjars studied by us are Muslim migrant shepherds who visit HP forest areas every year. They start migrating from Uttarakhand in April and reach HP in May every year. They again start migrating out from HP in the last week of September and reach Uttarakhand in October. Gujjars live in primitive and poor conditions primarily in kutcha hutments (thatched). They abandon their deras when they leave for Uttarakhand. Gujjars have reported to have seen few red colored and few white colored rats. They knew the site of rat burrows. They were aware of an outbreak in HP, 
which occurred in 2002 and spread to Uttar Pradesh (UP). They opined that body temperature shoots high in plague. They called it 'an disease of cold' (Thande ki Bimari).

It emerged through various FGDs and IDIs conducted by our research team that during outbreak people use to keep their mouth covered. The health workers were also of the view that exposure of people to plague was due to deep forest exploration for hunting. They told that even the doctors working in the hospitals started wearing double masks and the prophylactic treatment of all the health workers treating diseased patients during outbreak was started. All the new suspects were kept in special wards and all the precautionary measures were taken. Some respondents (doctors who were in service in 1980s) told that one plague outbreak also occurred in 1983 in Tangnu and Jaangli villages of Tehsil Chirgaon (pneumonic plague) and also in September 1984 in Mandol village (bubonic plague). They told that these were not reported in any documents of State as well as Central Government.

Overall 159 rodents and 22 fleas were collected from wild and domestic areas. Three species of rodents were identified: Rattus rattus ( $\mathrm{n}=132,83.01 \%$ ), Rattus norvegicus ( $\mathrm{n}=26,16.36 \%$ ) and Peromyscus leucopus ( $\mathrm{n}=1,0.63 \%)$. Similarly, three flea species namely Nosopsyllus fasciatus (Northern rat flea) ( $\mathrm{n}=11,50 \%)$, Epitedia wenmanni ( $\mathrm{n}=9,40.9 \%)$ and Leptophylla segnis (mouse flea) ( $n=1,4.5 \%)$ were extracted. Serum and blood samples $(n=49)$ collected from rodents showed Grampositive bacilli (GPB), Gram-positive cocci (GPC) and diphtheroid bacilli.

All the samples tested were serologically negative by RDT test kit.

\section{DISCUSSION}

Plague figures prominently among the list of re-emerging disease globally as well in India and continues to play havoc with the lives of the people. Thousands of cases still reported yearly to WHO indicates that plague is far from being eradicated and continues to present a threat, especially where public health and living conditions are poor. ${ }^{4,5}$ Many major epidemics have been reported in India, mostly linked with famine. Recurrent outbreaks of plague in India in the recent past have exposed not only the vulnerability of the Indian population to epidemics, but also the glaring lack of an efficient disease surveillance system.

Human encroachment on virgin forests keeps supplying us with new virulent diseases, the aspect which will be difficult to control. It is reported that the natives of plague affected belts of HP used to go for hunting in jungles during winter season. Simultaneously, they also indulge in collection of 'guchchi' a type of mushroom found in forest earn their livelihood. This way, possibly they usually come in contact with the wild rodents/animals that are infected with microbiological agents. These people often go in groups and stay in caves for days together. It has been reported that people can be infected directly from a plague infected rodent or other animal while handling, skinning or cutting up the meat. ${ }^{6}$

It is strongly believed by most of respondents who participated in 'FGD and IDI' that this disease repeats itself after every 10 to 15 years and outbreak could reoccur again somewhere around 2012 when heavy snowfall is predicted (by Devtas). Observations made in several areas of the world suggest that there is a cyclic periodicity of rodent plague which is generally reflected by the number of human cases peaking every 5 or 10 years. ${ }^{7}$ It is expected that natural foci will persist in caves and other difficult to access areas. So, HP should be kept on alert especially in heavy snowfall period.

The lifestyle conditions of Gujjars were also analyzed in the present study. They live deep in forests away from the mainland natives. They could, therefore be a potential link between any source of infection in forests and in native HP people. They have also been said to regularly visit the caves in deep forests. Therefore, they are regularly exposed to the microclimate of rats/cave, etc.

Because of lack of prior training, health care workers, laboratory personnel and other public health authorities in plague-endemic areas are not familiar with plague diagnosis and treatment. It is therefore important that its details should be revived in medical undergraduate teaching. A professional, scientific, area specific approach, considering behavioral/anthropological/socioepidemiological/environmental factors should be adopted to predict and tackle any subsequent outbreak of plague efficiently. Postgraduates in field of Community Medicine should undertake such operational topics for their dissertations so as to suggest the policy makers about designing future strategies. $^{8}$

There are about 220 species of rodents and 3,000 species of fleas present in the different habitat of India viz, mountain, plain, steppers, desert, cultivated field and forest in both temperate and tropical climate. All the rodent species are known to be infested with plague bacilli ( $Y$. pestis). Out of these, 31 species were proven as a vector of plague. $X$. cheopis, $X$. brasiliensis and Nosopsyllus fasciatus are the predominant rat flea species found generally in both domestic and peridomestic rodents. However, $X$. astia is normally found only on wild rodents. Among wild rodents, Tatera indica is the major natural reservoir of plague. There 
is a lack of sufficient rodent-vector literature involving relationship and the role of sylvatic cycle in plague causation.

The present study revealed that there was an intermixing (commingling) of animal host and their ectoparasites. It can be supported by the fact that Nosopsyllus fasciatus, a species normally found on domestic rodents, was also found on wild rodents. This confirms the exchange of flea hosts in commingling and sharing between $R$. rattus and Rattus norvegicus. This commingling can also allow $Y$. pestis to be maintained in both wild and domestic rodents and thus the increased chances of spreading of disease to man. Another study where $Y$. pestis found on both wild and domestic rodents reported that knowledge about flea populations on sylvatic and commensal animal hosts in specific locations may aid in forecasting the likelihood of plague. ${ }^{9}$

The given complexity of sylvatic cycle, including the number and variety of potential animal and vector hosts involved, it is unlikely that plague will ever be eradicated by human endeavors. Plague is not currently a prevalent human disease; it is thoroughly entrenched in widespared zoonotic loci that are unlikely to be eliminated. The recent epidemics in India remind us that its resurgence merely requires favorable environmental and public health conditions. The absence of new plague cases in recent past should not lead to complacence. This could also be due to 'silent period'. This may last as long as for 10 years or even longer. Thereafter, sudden explosions of rodent or human plague may occur (when favorable conditions are there). Therefore, the present study relating the vector-rodent related information may help in forecasting a plague outbreak in HP. The sylvatic cycle persisted in plague prone areas of HP as Nosopsyllus fasciatus, which is an efficient plague vector species was present in wild rodents, normally found on domestic and peridomestic rats. Therefore, regular surveillance for human, vector, animal and environmental is needed in upper hilly areas of HP.

So, plague control strategies need to be kept on alert and continually updated with the advances in biotechnology. In this context, diagnosis of plague is an important concern. This is because the initiation of appropriate control measures at proper time is only possible, if the disease is diagnosed in time. If the diagnosis is delayed, untreated and inappropriately treated cases of plague may spread the infection in general population. This actually happened in 2002 plague outbreak of HP. where the disease was diagnosed late and some other patients got infected leading to the spread of disease.

In the current scenario where quick public health response is the major concern, there should be modernistic approach for plague diagnosis. Use of RDT will prove crucial in this regard. In many other diseases like blood borne diseases viz. hepatitis B and C, HIV, syphilis, malaria and infectious diseases like typhoid, influenza, tuberculosis, dengue, etc. also RDTs are being routinely used. RDTs for plague detection are available and can be effective for helping manage such situations; cost-effective, easy to use, and reliable. It has also been proven to be rapid and easy to use at the bedside with almost $100 \%$ specificity and sensitivity. Importantly, detection of the F1-antigen is possible even in cases that have been previously treated, which is common in many patients in the outbreaks. Even though the RDT results have no confirmatory value during the early phase of the outbreak, they enable management of close contacts to be focused around cases defined as probable within 15 minutes. Notably, the RDT becomes a field confirmation test after $Y$. pestis has been identified on site. ${ }^{10}$

The results of our study confirm the fact that RDT kit is a reliable test. We tested 200 samples. The RDT results corresponded with bacteriological tests wherein, all these samples bacteriologically negative was also tested serologically negative with the RDT kit. But its validity need to be further tested and these kits should be used in India in the field conditions.

The research team is working in close collaboration with Plague Surveillance Unit at Civil Hospital, Rohru. The team is working toward generating awareness among plague affected villagers, Gujjars and health care staff and village natives about the causation, proneness of plague epidemics and control related factors. In addition, the research will help in understanding the local disease pattern and delineate high risk areas. Stress is being laid on taking preventive and containment measures with emphasis on reduction of man-vector contact in relation to plague to the State authorities to control the future plague outbreaks.

\section{REFERENCES}

1. Chamberlain NR. Sylvatic cycle in plague. Available from: ASMMicrobeLibrary.org@Chamberlain. Copyright 2003.

2. Erickson DL, Waterfield NR, Vadyvaloo V, Long D, Fischer ER, Ffrench-Constant R, et al. Acute oral toxicity of Yersinia pseudotuberculosis to fleas: Implications for the evolution of vector-borne transmission of plague. Cellular Microbiol 2007; 9(11):2658-66.

3. Aggarwal SP. Plague control in India. Directorate General Health Services. Ministry of Health and Family Welfare, 2005.

4. Sarah ER, Sean MR, Edward TR. Yersinia pestis and the plague. Am J Clin Pathol 2003;109(1):S78-S85.

5. WHO programmes and projects global alert and response, disease covered by EPR, Plague, 2009. Available form: http:// www.who.int/csr/en/. 
6. Joshi K, Thakur JS, Kumar R, Singh AJ, Ray P, Jain S, et al. Epidemiological features of pneumonic plague outbreak in Himachal Pradesh, India. Trans R Soc Trop Med Hyg 2009; 103(5):455-60.

7. Pan America Health Organization. Plague: Epidemiology and control. Epidemiological Bulletin, PAHO 1984;5(6):1-4.

8. Goel S, Singh A. Will plague continue to haunt hilly states of India? Int J Health 2008;8(1):1528-8315.

9. Eskey CR, Haas VH. Plague in the Western part of the United States. US Pub Health Bull 1940;25:83.

10. Bertherat E, Thullier P, Christophe JS, Kathleen E, Koné ML, Arntzen L, et al. Lessons learned about pneumonic plague diagnosis from two outbreaks, Democratic Republic of the Congo. Emerg Infect Dis 2011 May;17(5):778-84.

\section{ABOUT THE AUTHORS}

\section{Harvinder Kaur}

Research Associate, Department of Community Medicine, School of Public Health, Postgraduate Institute of Medical Education and Research, Chandigarh, India

\section{Sonu Goel (Corresponding Author)}

Assistant Professor of Health Management, Department of Community Medicine, School of Public Health, Postgraduate Institute of Medical Education and Research, Chandigarh, India, Phone: +91-172-2755215 09914208490, 9914208027, e-mail: sonugoel007@yahoo.co.in

\section{Yachna Sharma}

Junior Research Fellow, Department of Community Medicine, School of Public Health, Postgraduate Institute of Medical Education and Research, Chandigarh, India

\section{Rupali Raj Kessar}

Junior Research Fellow, Department of Community Medicine, School of Public Health, Postgraduate Institute of Medical Education and Research, Chandigarh, India

\section{Amarjeet Singh}

Professor, Department of Community Medicine, School of Public Health, Postgraduate Institute of Medical Education and Research Chandigarh, India 